\title{
A versatile method for bladder segmentation in computed tomography two-dimensional images under adverse conditions
}

\author{
João Ribeiro Pinto ${ }^{1}$ and João Manuel R. S. Tavares ${ }^{2}$
}

\begin{abstract}
This article presents the design and evaluation of an algorithm for urinary bladder segmentation in medical images, from contrastless CT studies of patients suffering from bladder wall tumours. These situations require versatile methods of segmentation, able to adapt to the structural changes the tumours provoke in the bladder wall, reflected as irregularities on the images obtained, creating adversities to the segmentation process. This semi-automatic method uses Fuzzy c-Means clustering, a Gaussian-curve-based intensity transformation, and Active Contour Models, requiring only the physician's input of a single seed point for each anatomical view, in order to segment the bladder volume in all frames that include it.

The performance of the method was evaluated on eight TCGA-BLCA collection patients, achieving approximately $79 \%$ of successful segmentations for small tumour patients (below $2.0 \mathrm{~cm}$ of diameter), and approximately $72 \%$ between 2.0 and $2.9 \mathrm{~cm}$. Successful segmentations for small tumour patients presented an average of $3.7 \mathrm{~mm}$ Hausdorff Distance and $91.0 \%$ Degree of Overlap. The promising performance attained, especially for small tumour patients, revealed a high potential of this method to serve as basis for an effective early-stage bladder wall tumour CAD system.
\end{abstract}

\section{Keywords}

Medical Imaging; Image Segmentation; Active Contours; Fuzzy c-Means; Urinary bladder; Cancer.

\section{Introduction}

According to the most recent statistics, concerning the year of 2015, urinary bladder cancer is the fifth most frequent type of cancer, responsible for almost 80,000 new cases and more than 15,000 deaths in the United States of America alone. ${ }^{1}$

Despite these worrying statistics, urinary bladder cancer is one of the most survivable types of cancer. In cases of early detection, with cancer still confined to the bladder, $94 \%$ of the patients have been successfully treated and survived the cancer. However, for late detections, the survival rate drops abysmally to $6 \%$. $^{2}$

This colossal difference calls for a correct and thorough examination of all cases where haematuria, the common and usually sole visible symptom, ${ }^{3 ; 4}$ presents itself, in order to ensure the detection of tumours before the cancer invades surrounding structures or metastasis takes place, and thus guarantee the greatest survival probability for the patient.

In this race against time, cystoscopy is the commonly performed, and usually required, exam. ${ }^{2-5}$ However, it presents many setbacks, as it is very limited, timeconsuming, invasive, and painful, ${ }^{4 ; 5}$ thus being currently supported or replaced by imaging technologies. ${ }^{6}$

Computed Tomography (CT) and Magnetic Resonance Imaging (MRI) are the usual choices. ${ }^{7}$ MRI is the most commonly used for the development of urinary bladder computer-aided-diagnosis (CAD) systems, as it provides good image resolution and soft tissue contrast, allowing easy distinction between them. ${ }^{6}$ However, CT is the imaging method most used by physicians for manual diagnosis and staging, due to the lower exam costs and much faster completion times, ${ }^{8}$ and because it provides higher patient comfort, despite the exposure to ionizing radiation. ${ }^{7}$

There is, thus, a lack of correspondence between the imaging modality commonly used for manual diagnosis by physicians, and the technology most of the CAD methods are prepared to use. Also, among the few CAD methods that use CT images, the large majority was tested solely in patients without cancer, or only with early-stage tumours, that do not influence greatly the overall image of the bladder and, thus, offer little extraordinary adversity to the segmentation process.

In this article, we present an enhancement of the FuzzySnake Segmentation algorithm, ${ }^{9}$ using Fuzzy c-Means clustering, a Gaussian-curve-based intensity transformation, and Active Contour Models (Snakes). The method was designed to segment the bladder in the axial, coronal, and sagital views of CT studies, aiming to be simple and versatile enough to adapt to various patients suffering from bladder wall cancer, with diverse tumour dimensions and locations.

\footnotetext{
${ }^{1}$ Faculdade de Engenharia, Universidade do Porto, Porto, Portugal 2 Instituto de Ciência e Inovação em Engenharia Mecânica e Engenharia Industrial, Departamento de Engenharia Mecânica, Faculdade de Engenharia, Universidade do Porto, Porto, Portugal

\section{Corresponding author:}

João Manuel R. S. Tavares, Faculdade de Engenharia da Universidade do Porto, R. Dr. Roberto Frias s/n, 4200-465 Porto, PORTUGAL

Email: tavares@fe.up.pt
} 
In the following sections of this article, a detailed outline of the method is exposed, along with the results and discussion of the performance evaluation, and a comparison with other commonly used algorithms for urinary bladder segmentation.

\section{Exploring common methods}

Considering the goal of precisely segmenting the urinary bladder in a plurality of adverse conditions, such as different patients, with different bladder sizes and shapes, and/or different tumour dimensions and locations, it stands to reason that the least complex methods should be the ones to effectively fit this generalization need.

Thus, the first step towards the development of the final method was exploring the most common segmentation methods, that present good results for a wide range of applications, including medical image processing and, specifically, bladder segmentation: Active Contour Models (Snakes), ${ }^{10-14}$ Region Growing, ${ }^{12 ; 15-17}$ and Chan-Vese Models. ${ }^{18-24}$

As the method developed, these algorithms were tested on eight patients of The TCGA Urothelial Bladder Carcinoma collection. ${ }^{25 ; 26}$ The wide variety of clinics, equipments, bladder cancer patients, and their characteristics, makes this collection of CT and MRI studies a great tool to adequately and thoroughly confirm the capabilities of the methods for bladder segmentation.

\section{Overview of the methods}

Active Contour Models ACM or, commonly, Snakes, ${ }^{27}$ are spline curves that iteratively move along the image, converging to regions of local energy minima. Energy is a combination of image energy, with a strong contribution from intensity gradient magnitude, that attracts the snake to edge regions, and of internal energy and external constraints, that control the evolution and smoothness of the contour. In this case, a circumference of 15 px radius centred in a user input seed point inside the bladder was used as initialization, and the parameters were optimized manually.

Region Growing One of the simplest methods of image segmentation, Region Growing (RG) uses seed points on the image that will partition the space in regions, by iteratively adding one neighbouring pixel to a region if they are similar enough in intensity. However, it has no smoothness or shape constraints for the contour. In this case, only one seed point was used, and the stopping criterion was the maximum intensity difference between the region and the neighbouring point $\epsilon=0.15$.

Chan-Vese Models Also called Active Contours Without Edges, ${ }^{28}$ the Chan-Vese method (CV) can be described as a combination of Region Growing and ACM. Like Region Growing, Chan-Vese Models also use region intensity similarities to grow the region being segmented, and do not rely on gradient computations as ACM. However, Chan-Vese Models also include area and perimeter regularizing terms that help enforce a degree of smoothness to the contour. Here, a $11 \times 11$ px square, centred on the already mentioned seed point, was used as initial mask for the segmentations.

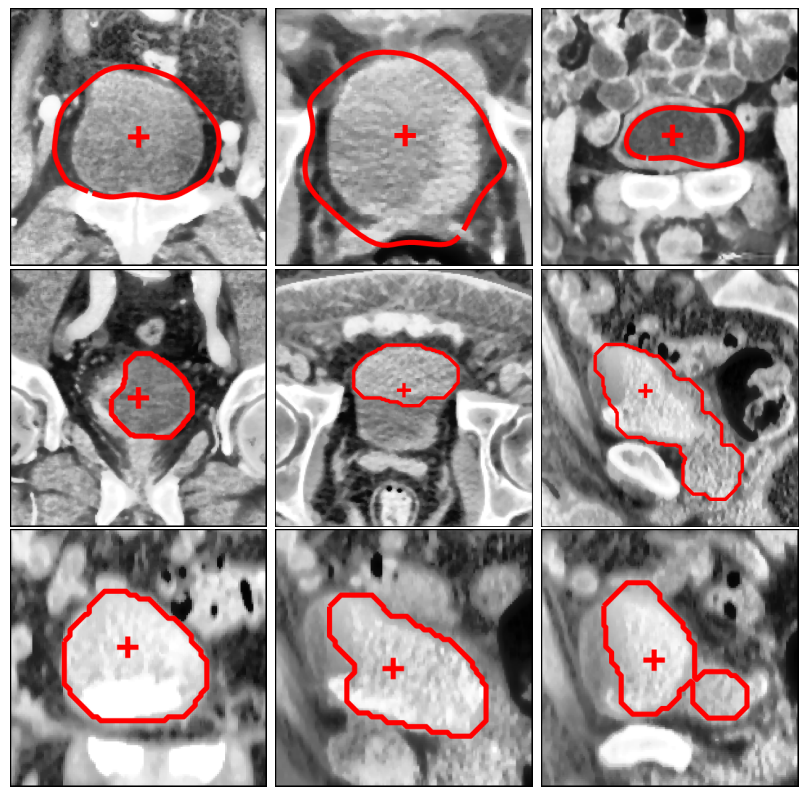

Figure 1. Examples of common results, which illustrate the behaviour of the three related methods (final segmentation contours (red lines) and seed point locations (red crosses); first row: ACM results; second row: Region Growing results; third row: Chan-Vese Model results).

\section{Common Results}

From the application of the three related methods to the images under studied, some patterns of behaviour could be observed (Fig. 1).

With ACM, it was common to observe an overwhelming attraction of the contour to bone edges instead of bladder edges, as the former generally has much higher gradient magnitude due to bone's maximum intensity on the images. Also, when the bladder wall is much more intense than the bladder lumen, it is frequent for the snake to segment the lumen only.

With Region Growing, due to the lack of contour smoothness constraints, it was frequent to obtain irregular contours and, due to being based on intensity similarities, higher-intensity tumours and darker regions of the bladder lumen were frequently left out of the segmented region, while, in other cases, leakages to similar neighbouring structures was also frequent.

Finally, Chan-Vese Model presented better results, reducing the influence of bone edges and intensity differences, avoiding the occurrence of leakages, and producing smooth contours. However, the contours rarely fit the true edge of the bladder and, instead, usually fell short.

Analysing these results, it was possible to conclude that, the better results of Chan-Vese Model denote that the likely path to solve this problem is combining the region properties of Region Growing and the smoothness of ACM. However, a refined method must be developed in order to obtain better segmentation performance. 


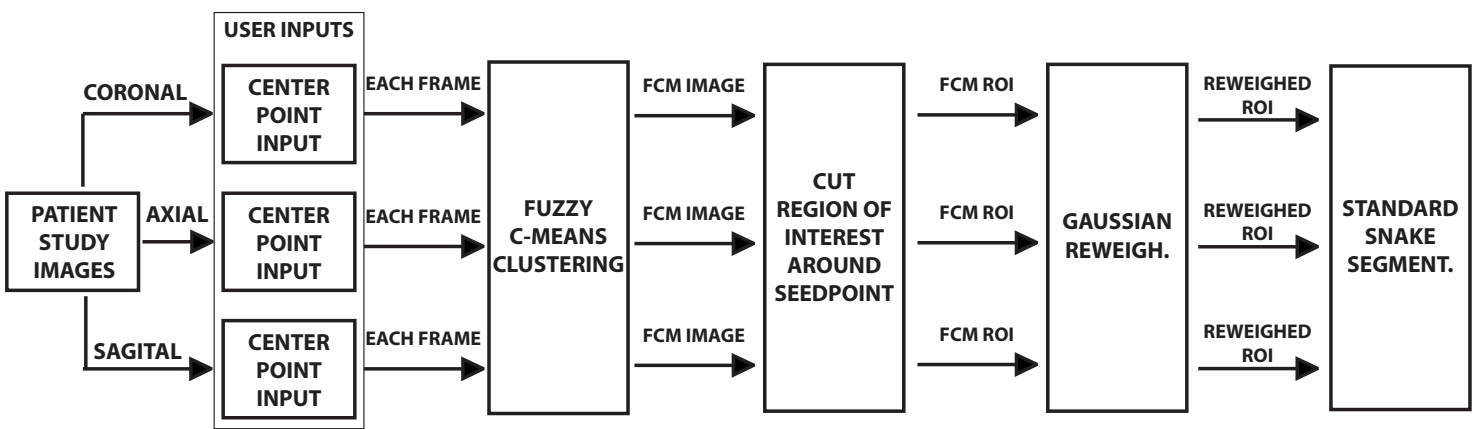

Figure 2. Outline of the method.

\section{The approach}

\section{Structure and overview}

The method developed consisted in an enhancement of the Fuzzy-Snake method, proposed by Bueno et al. ${ }^{9}$ The FuzzySnake method was built around the same objective as this one, $i$. $e$. to segment the urinary bladder in contrastless CT images. Despite presenting results for a large variety of cancer patients, no indication is given on whether the patients' tumours are in the bladder (that could influence greatly the segmentation results), or whether the method was tested for images of anatomical views other than axial.

Our improved method consisted on five steps (Fig. 2). First, for each view (axial, coronal, and sagital), a single seed point, roughly in the centre of the bladder area is selected on the middle frame (where the bladder is larger). This seed point is to be used as reference for some following procedures, as the centre for the cropping of the region of interest, and as the centre for the mode computation before the Gaussian reweighing. The method benefits from the selection of the seed point as close as possible to the centre of the bladder area; however, it was developed to be flexible to variations.

Afterwards, a fuzzy c-means clustering is applied to each image under study, the image region of interest is cropped and an intensity transformation based in a Gaussian curve is applied, and a final segmentation is obtained using ACM. The details of each of these steps are presented in the following sections.
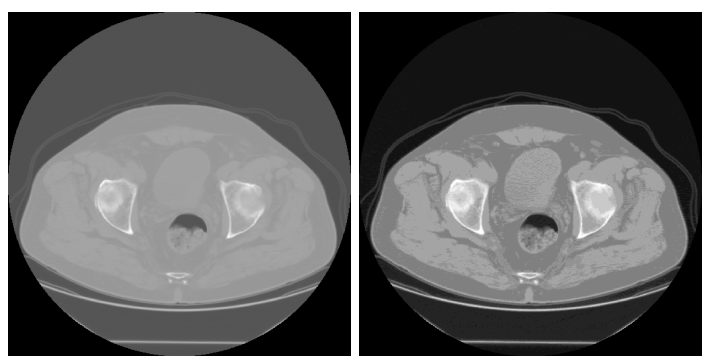

Figure 3. Example of the application of the Fuzzy c-Means clustering algorithm, with $c=15$ (left: original image; right: FCM image).

\section{Fuzzy c-means clustering}

Fuzzy c-Means (FCM) clustering is the designation given to an algorithm first developed by Dunn, ${ }^{29}$ and later improved by Bezdek et al., ${ }^{30 ; 31}$ based on the widely used k-Means method. ${ }^{32}$ FCM is an unsupervised clustering method used to group data points according to the similarity between their attributes.

In images, the FCM algorithm clusters their pixels according to their intensity, into $c$ clusters. In each iteration, the algorithm computes the centroid of each cluster, and determines a measure of membership to each cluster for each point, according to the similarity of the pixel's and centroid's intensities. Thus, the Fuzzy c-Means algorithm iteratively approximates to the minimum of its goal function, defined as:

$$
J(U, V)=\sum_{i=1}^{c} \sum_{k=1}^{N}\left(u_{i k}\right)^{m}\left\|x_{k}-\nu_{i}\right\|^{2} .
$$

where $c$ and $N$ represent, respectively, the number of clusters and the number of pixels, $U$ the set of membership values $u_{i k}$, and $v$ the set of cluster centroids $\nu_{i}$. The values of $u_{i k}$ and $\nu_{i}$ are computed through the expressions:

$$
\begin{gathered}
u_{i k}=\left[\sum_{j=1}^{c}\left(\frac{\left\|x_{k}-\nu_{i}\right\|}{\left\|x_{k}-\nu_{j}\right\|}\right)^{\frac{2}{m-1}}\right]^{-1}, \forall i, k . \\
\nu_{i}=\frac{\sum_{k=1}^{N}\left(u_{i k}\right)^{m} \cdot x_{k}}{\sum_{k=1}^{N}\left(u_{i k}\right)^{m}}, \forall i, k
\end{gathered}
$$

The algorithm comes to a stop when it reaches a set limit of iterations, or when the sum of the centroids norm variation between iterations is lower than a defined threshold $\epsilon$ :

$$
E_{i}=\sum_{i=1}^{c}\left\|\nu_{i, t+1}-\nu_{i, t}\right\|<\epsilon .
$$

Each pixel, in the end, joins the cluster that corresponds to its highest measure of membership. As proposed by Bueno et al., ${ }^{9}$ and confirmed though the experimentation with $c$ between 9 and 20, the number of clusters was defined as 15 . The labels obtained were ordered according to the intensity of the image regions they were attributed to, and the combination of this with the number of clusters allowed to obtain a thorough separation of the different tissues in each image into different clusters (see Fig. 3), each being attributed a different label. This separation 
is enhanced by the Gaussian reweighing, that effectively consists on a transformation of the label ordering, that will be essential to maximise the performance of the subsequent ACM segmentation. The FCM application also effectively performs a histogram stretch on the image, and reduces intracluster noise and variations, eliminating the need for image pre-processing.

\section{Cropping of the region of interest}

In order to obtain a correct computation of the cluster centroids and measures of membership, the FCM method requires the entire image as input. However, for the following steps, this is not necessary, and is even undesirable, as performing segmentation or other image processing computations in entire images can be time consuming.

Thus, each square image, after FCM, is cropped into a square region of interest (ROI), centred on the seed point, with side length $L_{R O I}=L_{I} / 3$, where $L_{I}$ is the side length of the original image (Fig. 4).

This step ensures the reduction of the total number of pixels to a ninth of the original, effectively reducing the time needed to perform the following computations. In all images used for testing the method, the whole bladder region was consistently inside the ROI, independently of the seed point location inside it, even when considering seed point selection in the extremities of the bladder region; and it thus expected to correctly include the whole bladder in images of any patient, and be flexible to variable seed point locations.

\section{Gaussian reweighing}

After the cropping of the ROI, the labels of each pixel, originated by the FCM on the first step, had to be transformed into intensity values.

This step was not included in the original FuzzySnake method. Instead, the ACM used by Bueno et al. ${ }^{9}$ was modified in order to receive both the original and FCM images. In this case, where we want to preserve the generalization capabilities of the method, this modification could undermine our objective. Thus, the original formulation of ACM is used.

To the FCM image (with label $[1,15]$ for each pixel) is applied an intensity transformation based on a Gaussian curve (Fig. 4). The mode $m$ of the labels of a square $5 \times 5$ px neighbourhood centred on the seed point is determined, representing the main label of the bladder region, and is used as mean of a Gaussian curve with $\sigma=0.5$. The new intensity value $y_{i}$ of each pixel, according to its FCM label $x_{i}$, is then
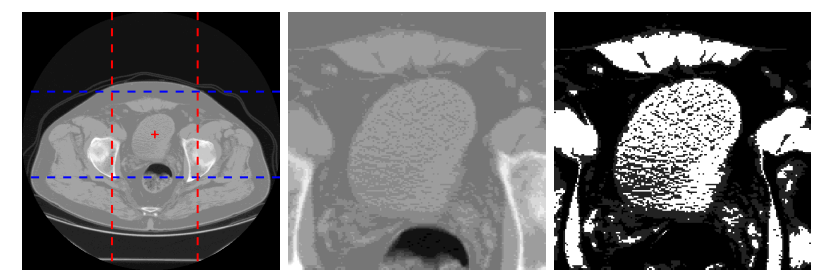

Figure 4. Example of the cropping of the ROI and Gaussian reweighing (left: FCM image with seed point (red cross) and the limit lines of the ROI; center: cropped ROI; right: reweighed image).

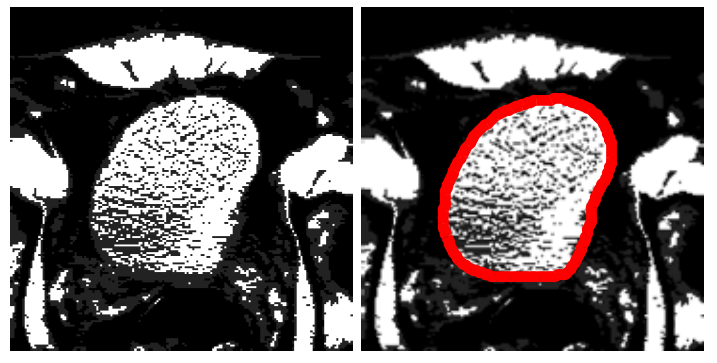

Figure 5. Example of the application of ACM after FCM and Gaussian reweighing. (left: reweighed image; right: ACM segmentation result). The usefulness of ACM in ignoring the noise inside the bladder region and fitting the true bladder contour is visible.

computed through the expression:

$$
y_{i}=e^{\frac{-\left(x_{i}-m\right)^{2}}{2 \sigma^{2}}} .
$$

This transformation is used mainly to increase the intensity difference between the clusters with labels close to the mode $m$, weighing the bladder region with the highest intensity, and thus enhancing the gradient magnitude on the bladder edge, and highly attenuating the influence of bone edges and other different structures on the ACM that follows.

\section{Active contour model segmentation}

The last step is the segmentation of the reweighed ROI. Active Contour Models (ACM, commonly designated as Snakes), were first described by Kass et al., ${ }^{27}$ and were ever since praised by their usefulness and applicability in multiple computer vision applications.

ACM are spline curves $\nu(s)=(x(s), y(s))$, that progress iteratively under the influence of image forces $\left(E_{\text {image }}\right)$, external constraint forces $\left(E_{\text {external }}\right)$, and internal forces ( $\left.E_{\text {internal }}\right)$, until converging to locations of energy minima. The overall energy is defined by:

$$
\begin{aligned}
E_{\text {snake }}= & \int_{0}^{1}\left[E_{\text {internal }}(\nu(s))++E_{\text {image }}(\nu(s))\right. \\
& \left.+E_{\text {external }}(\nu(s))\right] d s .
\end{aligned}
$$

The internal energy depends on the mechanical properties of the spline, through its first and second order derivatives, weighted using the $\alpha$ and $\beta$ parameters, with:

$$
E_{\text {internal }}=\left(\frac{\alpha(s)}{2}\left|\nu_{s}(s)\right|^{2}+\frac{\beta(s)}{2}\left|\nu_{s s}(s)\right|^{2}\right) .
$$

The image energy is computed through a weighted combination of energy functionals, relative to the energy of lines, edges, and line terminations on the image, through the expression:

$$
\begin{aligned}
E_{\text {image }}= & \left(w_{\text {line }} E_{\text {line }}+w_{\text {edge }} E_{\text {edge }}\right. \\
& \left.+w_{\text {term }} E_{\text {term }}\right)
\end{aligned}
$$

where the line, edge, and line termination energy are computed through:

$$
E_{\text {line }}=I(x, y),
$$


Table 1. Subjective evaluation of segmentations by patient tumour dimensions (T.S. - tumour diameter, N.F. - total number of frames, S.S. - number of successful segmentations).

\begin{tabular}{c|ccc}
\hline T.S. $(\mathrm{cm})$ & N.F. & S.S. & Success Rate (\%) \\
\hline \hline $0-1.9$ & 155 & 123 & 79.4 \\
$2.0-2.9$ & 129 & 93 & 72.1 \\
$\geqslant 3.0$ & 206 & 113 & 54.9 \\
\hline & $E_{\text {edge }}=-|\nabla I(x, y)|^{2}$, and \\
$E_{\text {term }}=\frac{C_{y y} C_{x}{ }^{2}-2 C_{y y} C_{x} C_{y}+C_{x x}+C_{y}{ }^{2}}{\left(C_{x}{ }^{2}+C_{y}{ }^{2}\right)^{3 / 2}}$,
\end{tabular}

where $C(x, y)=G_{\sigma}(x, y) * I(x, y)$ is a slightly smoothed version of the image.

Here, a circumference of $15 \mathrm{px}$ radius centred on the seed point is used as initialization, and grew to fit the highest gradient region (now corresponding to the edge of the bladder). To preserve generalization capabilities, the default parameters of the snake toolbox used ${ }^{33}$ were mostly maintained, with $\alpha=\beta=0.2, \quad k=2.0, \quad w_{\text {line }}=0.04$, $w_{\text {edge }}=2.0, w_{\text {term }}=0.01, \sigma_{1}=\sigma_{2}=2.0$, and without Gradient Vector Flow optimization.

The ACM allows to obtain a smooth boundary of the bladder region, fitted to the true contours of the bladder, and resistant to possible noise inside this region that may remain after FCM and the reweighing (Fig. 5).

\section{Results and discussion}

The algorithm was tested on all frames, individually, of the three views (axial, coronal, and sagital), of contrastless CT studies of eight different patients of the TCGA-BLCA collection. ${ }^{25 ; 26}$ These studies were chosen randomly, among the available variety, as long as they included contrastless CT images spanning the entire bladder, on all three anatomical views.

The studied TCGA-BLCA collection posed an interesting challenge (and opportunity) for this approach. Due to the large plurality of patients characteristics, equipment specificities, and acquisition settings, it increases the adversities that are be encountered by the method on its goal to segment the bladder, and effectively ensures it can be applied in a wide variety of contexts.

In order to obtain both extensive and rigorous measures of performance, the results were evaluated both subjectively and objectively. The location and dimensions of the bladder wall tumours were determined, for comparison purposes, and ground-truths of the frames used in the objective evaluation were obtained from an expert.

\section{Subjective performance assessment}

All segmentations performed were evaluated subjectively, and classified as 'Successful' or 'Unsuccessful'. The former was reserved to the segmentations that presented a final contour that adapted to the true bladder boundary in all of its extension, while the latter was attributed to the remainder ons. The evaluation results are presented in Table 1 , where the success rate is the percentage of 'Successful' segmentations performed.
The highest success rates were obtained for patients with smaller tumours, with almost $80 \%$ for less than $2.0 \mathrm{~cm}$ of diameter, while, for tumours greater than $3.0 \mathrm{~cm}$, the success rate of the method dropped to approximately $55 \%$. The segmentations obtained through the proposed method were also compared with the ones produced by ACM, RegionGrowing, and Chan-Vese Model. The new method proved to be much more efficient at fitting the true edge of the bladder (see Fig. 6 for some examples), while Region Growing and Chan-Vese usually caused leakages and/or left certain areas out, and ACM did not adapt to the true contour of the bladder and deviated to higher gradient zones.

For larger tumour patients, this size proved to be the method's main cause of failure, with the tumour frequently being left out of the segmented region. However, this was rarely observed in frames with tumours smaller than $2.0 \mathrm{~cm}$. Other causes of failure, independent of the tumour size, were encountered, such as the incapacity to adapt to the smaller bladder area in the few first and last frames of each view, and, more rarely, the effect of neighbouring structures on the snake contour.

\section{Objective performance assessment}

Having the rates of successful segmentations determined through a subjective evaluation, two random sample segmentation results for each view, one classified as successful and the other as unsuccessful, were selected for

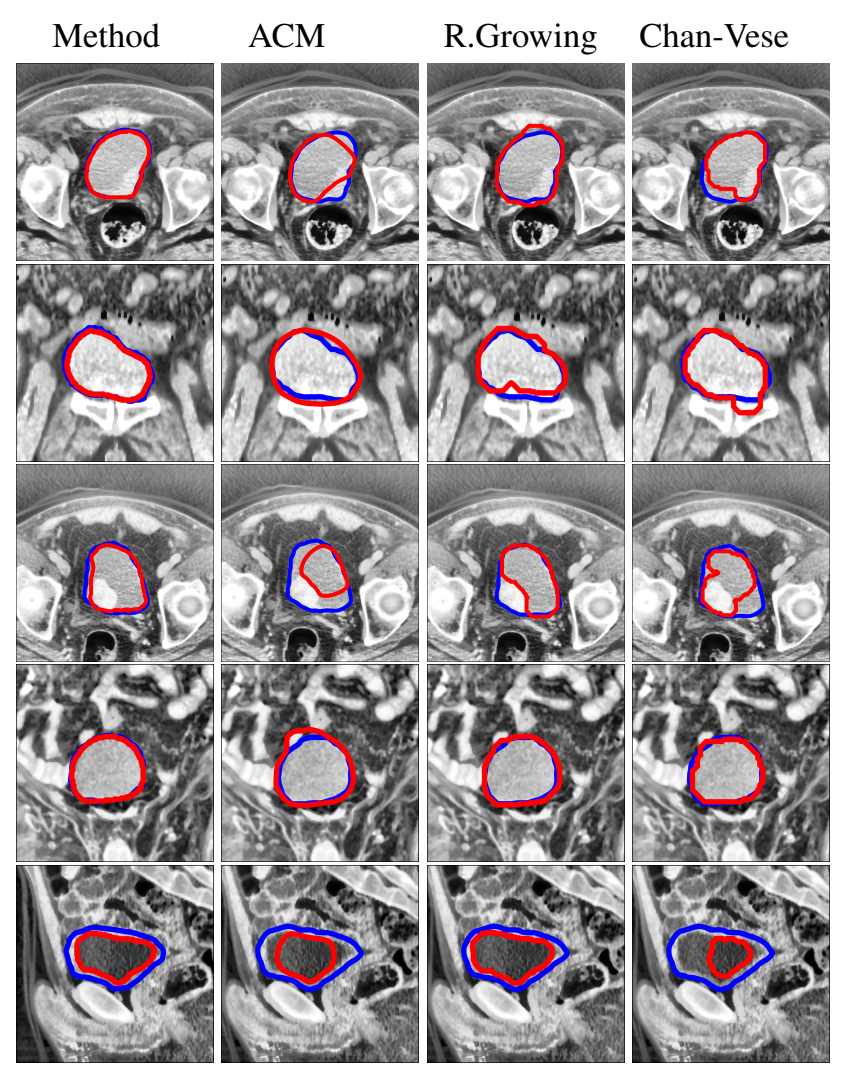

Figure 6. Comparison of some results obtained by the proposed method and the common methods explored, for images belonging to subjects with tumours of different dimensions (red contour: segmentation result, blue contour: ground-truth; tumour sizes, from first to last row: $1.2 \mathrm{~cm}, 1.2 \mathrm{~cm}$, $2.7 \mathrm{~cm}, 3.1 \mathrm{~cm}$, and $3.2 \mathrm{~cm}$ ). 
Table 2. Objective evaluation of segmentations by patient tumour dimensions, for the sample successful segmentations (T.S. - tumour diameter, N.F. - number of frames evaluated, HD Hausdorff Distance, OL - Degree of Overlap).

\begin{tabular}{c|ccc}
\hline & \multicolumn{3}{|c}{ Successful Segmentations } \\
T.S. $(\mathrm{cm})$ & N.F. & HD $(\mathrm{mm})$ & OL $(\%)$ \\
\hline \hline $0-1.9$ & 9 & $3.7 \pm 0.9$ & $91.0 \pm 4.4$ \\
$2.0-2.9$ & 6 & $5.7 \pm 2.4$ & $84.9 \pm 4.1$ \\
$\geqslant 3.0$ & 9 & $6.1 \pm 2.8$ & $85.4 \pm 10.3$ \\
\hline
\end{tabular}

Table 3. Objective evaluation of segmentations by patient tumour dimensions, for the sample unsuccessful segmentations (T.S. - tumour diameter, N.F. - number of frames evaluated, HD Hausdorff Distance, OL - Degree of Overlap).

\begin{tabular}{c|ccc}
\hline & \multicolumn{3}{|c}{ Unsuccessful Segmentations } \\
T.S. $(\mathrm{cm})$ & N.F. & HD $(\mathrm{mm})$ & OL $(\%)$ \\
\hline \hline $0-1.9$ & 9 & $19.3 \pm 8.7$ & $70.3 \pm 10.9$ \\
$2.0-2.9$ & 6 & $19.5 \pm 3.6$ & $62.2 \pm 11.4$ \\
$\geqslant 3.0$ & 9 & $22.7 \pm 5.8$ & $65.5 \pm 11.8$ \\
\hline
\end{tabular}

each patient, and compared to the ground truth using two metrics: Hausdorff Distance (HD) and Degree of Overlap (OL, using the Jaccard similarity index) (Tables 2 and 3).

The best results were found, as expected, for patients with smaller tumours, with the method presenting similar performance to that of the original Fuzzy-Snake method for tumours under $2.0 \mathrm{~cm}$ of diameter $(91.0 \% \mathrm{OL})$, as well as a reduced Hausdorff Distance, that proves the adequacy of the segmentation contour to the ground-truth. For successful segmentations, the average results were always above $84 \%$ OL and below $7 \mathrm{~mm}$ HD. For the unsuccessful segmentations (the less common situations), the HD and OL values were, respectively, around 19-23 $\mathrm{mm}$ and 60-70\%, which generally corresponded to the size of the tumour region on the frames that, when left out, was the most common source of unsuccessful segmentations.

In Tables 4 and 5, the results of the evaluation according to the anatomical view are presented, in order to allow for the assessment of the method's performance in each view. As expected, for images with larger tumours, the performance is worse. The method seems to work better with axial images,

Table 4. Objective evaluation of segmentations by patient tumour dimensions and anatomical view, for the sample successful segmentations (T.S. - tumour diameter, HD Hausdorff Distance, OL - Degree of Overlap).

\begin{tabular}{cc|cc}
\hline & T.S. $(\mathrm{cm})$ & HD $(\mathrm{mm})$ & OL $(\%)$ \\
\hline \hline Axial & $0-1.9$ & $3.3 \pm 1.1$ & $94.3 \pm 1.7$ \\
& $2.0-2.9$ & $6.2 \pm 2.8$ & $87.8 \pm 5.0$ \\
& $\geqslant 3.0$ & $4.8 \pm 3.9$ & $91.0 \pm 5.3$ \\
\hline Coronal & $0-1.9$ & $3.5 \pm 0.1$ & $90.9 \pm 2.8$ \\
& $2.0-2.9$ & $4.1 \pm 0.6$ & $85.0 \pm 0.1$ \\
& $\geqslant 3.0$ & $5.8 \pm 2.7$ & $88.4 \pm 5.8$ \\
\hline Sagital & $0-1.9$ & $4.3 \pm 1.2$ & $87.6 \pm 5.8$ \\
& $2.0-2.9$ & $6.8 \pm 3.5$ & $81.8 \pm 4.9$ \\
& $\geqslant 3.0$ & $7.8 \pm 1.2$ & $76.7 \pm 13.7$ \\
\hline Overall & $0-1.9$ & $3.7 \pm 0.9$ & $91.0 \pm 4.4$ \\
& $2.0-2.9$ & $5.7 \pm 2.4$ & $84.9 \pm 4.1$ \\
& $\geqslant 3.0$ & $6.1 \pm 2.8$ & $85.4 \pm 10.3$ \\
\hline
\end{tabular}

Table 5. Objective evaluation of segmentations by patient tumour dimensions and anatomical view, for the sample unsuccessful segmentations (T.S. - tumour diameter, HD Hausdorff Distance, OL - Degree of Overlap).

\begin{tabular}{cc|cc}
\hline & T.S. $(\mathrm{cm})$ & HD $(\mathrm{mm})$ & OL $(\%)$ \\
\hline \hline Axial & $0-1.9$ & $18.0 \pm 9.0$ & $72.6 \pm 10.8$ \\
& $2.0-2.9$ & $18.9 \pm 0.2$ & $64.3 \pm 5.7$ \\
& $\geqslant 3.0$ & $27.2 \pm 4.9$ & $71.0 \pm 14.2$ \\
\hline Coronal & $0-1.9$ & $15.8 \pm 4.0$ & $76.1 \pm 8.6$ \\
& $2.0-2.9$ & $18.8 \pm 6.6$ & $64.3 \pm 4.9$ \\
& $\geqslant 3.0$ & $22.1 \pm 3.8$ & $58.4 \pm 7.7$ \\
\hline Sagital & $0-1.9$ & $24.2 \pm 12.1$ & $62.3 \pm 11.7$ \\
& $2.0-2.9$ & $20.8 \pm 4.0$ & $58.1 \pm 23.4$ \\
& $\geqslant 3.0$ & $18.9 \pm 6.7$ & $67.0 \pm 13.1$ \\
\hline Overall & $0-1.9$ & $19.3 \pm 8.7$ & $70.3 \pm 10.9$ \\
& $2.0-2.9$ & $19.5 \pm 3.6$ & $62.2 \pm 11.4$ \\
& $\geqslant 3.0$ & $22.7 \pm 5.8$ & $65.5 \pm 11.8$ \\
\hline
\end{tabular}

but this may be a result of the higher spatial resolution of these images versus the other views. The proposed method is also compared with ACM, RG, and CV methods (cf. Table 6), showing significantly improvements in all cases.

\section{Conclusions}

To conclude, the proposed method has overcome the difficulties associated with the task at hand, and successfully segment the urinary bladder using the most common imaging technology used in clinics, the contrastless computed tomography, while effectively maintaining the simplicity needed to generally fit the wide range of characteristics of different bladder wall tumour patients.

The evaluation results of the proposed method, both subjective and objective, strongly suggest its use as basis for a reliable CAD system. Along with an equally effective inner wall segmentation method, and perhaps tridimensional reconstruction for visualization purposes, the method could be the key to help bladder cancer diagnosis, especially for early-stage tumours.

Table 6. Objective evaluation of segmentations by patient tumour dimensions, and comparison with results of ACM, Region Growing, and Chan-Vese (T.S. - tumour diameter, HD Hausdorff Distance, OL - Degree of Overlap; best results obtained for each metric and each tumour size are presented in bold)

\begin{tabular}{cc|cc}
\hline & T.S. $(\mathrm{cm})$ & HD $(\mathrm{mm})$ & OL $(\%)$ \\
\hline \hline This method & $0-1.9$ & $\mathbf{3 . 7} \pm \mathbf{0 . 9}$ & $\mathbf{9 1 . 0} \pm \mathbf{4 . 4}$ \\
& $2.0-2.9$ & $\mathbf{5 . 7} \pm \mathbf{2 . 4}$ & $\mathbf{8 4 . 9} \pm \mathbf{4 . 1}$ \\
& $\geqslant 3.0$ & $\mathbf{6 . 1} \pm \mathbf{2 . 8}$ & $\mathbf{8 5 . 4} \pm \mathbf{1 0 . 3}$ \\
\hline ACM & $0-1.9$ & $11.9 \pm 4.9$ & $79.2 \pm 11.6$ \\
& $2.0-2.9$ & $17.8 \pm 7.9$ & $59.6 \pm 14.1$ \\
& $\geqslant 3.0$ & $16.1 \pm 8.0$ & $66.9 \pm 13.9$ \\
\hline R. Growing & $0-1.9$ & $7.1 \pm 3.6$ & $89.4 \pm 4.7$ \\
& $2.0-2.9$ & $16.0 \pm 13.9$ & $80.0 \pm 10.9$ \\
& $\geqslant 3.0$ & $13.5 \pm 10.0$ & $72.5 \pm 17.4$ \\
\hline Chan-Vese & $0-1.9$ & $12.4 \pm 10.2$ & $80.8 \pm 10.7$ \\
& $2.0-2.9$ & $24.9 \pm 3.9$ & $70.1 \pm 1.6$ \\
& $\geqslant 3.0$ & $22.0 \pm 14.3$ & $55.7 \pm 32.3$ \\
\hline
\end{tabular}


Future work possibilities include the evaluation of the proposed method with a larger number of studies, the reconstruction of the segmentations to obtain tridimensional models of the bladder, and the use of the method directly in $3 \mathrm{D}$, merging the three views in one single process, and comparing its performance to the method proposed in this article.

\section{Acknowledgements}

Authors gratefully acknowledge the funding of Project NORTE-01-0145-FEDER-000022 - SciTech - Science and Technology for Competitive and Sustainable Industries, cofinanced by "Programa Operacional Regional do Norte" (NORTE2020), through "Fundo Europeu de Desenvolvimento Regional" (FEDER).

The authors also acknowledge the effort of building and maintaining The Cancer Genome Atlas Urothelial Bladder Carcinoma (TCGA-BLCA) collection, and the individuals and institutions that have provided the data, and allowed its use on this project. It proved to be of inestimable worth for the development and evaluation of the proposed method.

\section{References}

1. Siegel RL, Miller KD and Jemal A. Cancer Statistics. $C A$ Cancer J Clin 2016; 66: 7-30.

2. Tate P. Seeley's Principles of Anatomy and Physiology. Second ed. McGraw Hill, 2012. ISBN 9780073378190.

3. Amling CL. Diagnosis and Management of Superficial Bladder Cancer. Curr Probl Cancer 2001; 25(4): 219-278.

4. Stamatiou K, Papadoliopoulos I, Dahanis S et al. The accuracy of ultrasonography in the diagnosis of superficial bladder tumors in patients presenting with hematuria. Ann Saudi Med 2009; 29(2): 134-137.

5. Jaume S, Ferrant M, Macq B et al. Tumor Detection in the Bladder Wall with a Measurement of Abnormal Thickness in CT Scans. IEEE Trans Biomed Eng 2003; 50(3): 383-390.

6. McKibben MJ and Woods ME. Preoperative imaging for staging bladder cancer. Curr Urol Rep 2015; 16(4): 1-7.

7. Hafeez $\mathrm{S}$ and Huddart R. Advances in bladder cancer imaging. BMC Med 2013; 11(104): 1-10.

8. Barentsz JO, Jager GJ, Witjes JA et al. Primary staging of urinary bladder carcinoma: the role of MRI and a comparison with CT. Eur Radiol 1996; 6: 129-133.

9. Bueno G, Martnez-Albal A and Adn A. Fuzzy-Snake Segmentation of Anatomical Structures Applied to CT Images. In Campilho A and Kamel M (eds.) International Conference on Image Analysis and Recognition ICIAR 2004 Part II. Springer-Verlag Berlin Heidelberg, 2004. pp. 33-42.

10. Fresno MD, Vnere $M$ and Clausse A. A combined region growing and deformable model method for extraction of closed surfaces in 3D CT and MRI scans. Comput Med Imaging Graph 2009; 33(5): 369-376.

11. Ivins $\mathrm{J}$ and Porrill J. Active region models for segmenting medical images. IEEE Image Proc 1994; 2: 227-231.

12. Ma Z, Jorge RN, Mascarenhas T et al. Novel Approach to Segment the Inner and Outer Boundaries of the Bladder Wall in T2-Weighted Magnetic Resonance Images. Ann Biomed Eng 2011; 39(8): 2287-2297.
13. Wang G, Xu J, Dong Q et al. Active Contour Model Coupling with Higher Order Diffusion for Medical Image Segmentation. International Journal of Biomedical Imaging 2014; 2014: 8.

14. Sethi G and Saini BS. Segmentation of abdomen diseases using active contour models in CT images. Biomedical Engineering: Applications, Basis and Communications 2015; 27(05): 1550047.

15. Pohle $\mathrm{R}$ and Toennis KD. Segmentation of medical images using adaptive region growing. In Sonka $\mathrm{M}$ and Hanson KM (eds.) Proc. SPIE, volume 4322. SPIE, 2001. pp. 1337-1346.

16. Rai $\mathrm{H}$ and Nair TG. Gradient Based Seeded Region Grow Method for CT Angiographic Image segmentation. Inter JRI 2009; 1: 1-6.

17. Navratnam S, Raman V, Fazilah S et al. Proposed Seed Pixel Region Growing Segmentation and Artificial Neural Network Classifier for Detecting the Renal Calculi in Ultrasound Images for Urologist Decisions. IJCSI 2016; 13.5: 62-68.

18. Ma Z, Tavares JMR, Jorge RN et al. A review of algorithms for medical image segmentation and their applications to the female pelvic cavity. Comput Methods Biomech Biomed Eng 2010; 13: 235-246.

19. Zhu H, Duan C, Jiang R et al. Computer-aided detection of bladder tumors based on the thickness mapping of bladder wall in MR images. In Dawant BM and Haynor DR (eds.) Proc. SPIE, volume 7623. SPIE, 2010.

20. Qin X, Lu H, Tian Y et al. Partial sparse shape constrained sector-driven bladder wall segmentation. Machine Vision and Applications 2015; 26(5): 593-606.

21. Chen L, Zhou Z and Wang J. Su-c-207b-03: A geometrical constrained chan-vese based tumor segmentation scheme for pet. Medical Physics 2016; 43(6): 3330-3330.

22. Pratondo $\mathrm{A}$, Chui $\mathrm{CK}$ and Ong SH. Integrating machine learning with region-based active contour models in medical image segmentation. Journal of Visual Communication and Image Representation 2017; 43: 1 - 9.

23. Cha KH, Hadjiiski LM, Samala RK et al. Comparison of bladder segmentation using deep-learning convolutional neural network with and without level sets. Proc SPIE 2016; 9785: 978512-978512-7.

24. Duan C, Liang Z, Bao S et al. A coupled level set framework for bladder wall segmentation with application to mr cystography. IEEE Transactions on Medical Imaging 2010; 29(3): 903-915.

25. Kirk S, Lee Y, Lucchesi FR et al. Radiology Data from The Cancer Genome Atlas Urothelial Bladder Carcinoma [TCGABLCA] collection, 2016. URL http://doi.org/10. 7937/K9/TCIA.2016.8LNG8XDR.

26. Clark K, Vendt B, Smith K et al. The Cancer Imaging Archive (TCIA): Maintaining and Operating a Public Information Repository. J Digit Imaging 2013; 26(6): 1045-1057.

27. Kass M, Witkin A and Terzopoulos D. Snakes: Active Contour Models. Int J Comput Vision 1988; 1: 321-331.

28. Chan TF and Vese LA. Active Contours Without Edges. IEEE Trans Image Process 2001; 10(2): 266-277.

29. Dunn JC. A Fuzzy Relative of the ISODATA Process and Its Use in Detecting Compact Well-Separated Clusters. J Cybernetics 1973; 3(3): 32-57.

30. Bezdek JC. Pattern Recognition with Fuzzy Objective Function Algorithms. First ed. Plenum Press, 1981. ISBN 978-1-47570452-5. 
31. Bezdek JC, Ehrlich R and Full W. FCM: The Fuzzy c-Means Clustering Algorithm. Comput Geosci 1984; 10(2-3): 191-203.

32. MacQueen J. Some Methods for Classification and Analysis of Multivariate Observations. In LeCam L and Neyman J (eds.) Proceedings of the Fifth Berkeley Symposium on Mathematical Statistics and Probability, volume 1. University of California Press, 1967. pp. 281-297.

33. Kroon DJ. Snake: Active Contour, 2010. URL http: / / www . mathworks.com/matlabcentral/fileexchange/ 28149-snake---active-contour. 